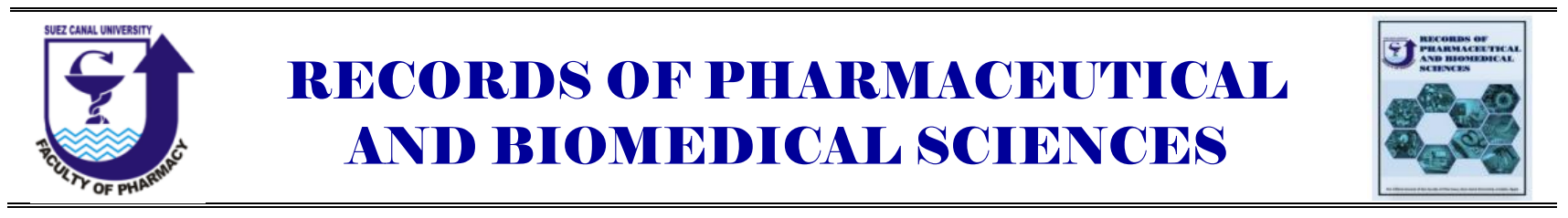

\title{
A review article on Ciprofloxacin determination with various analytical techniques
}

\author{
Menna El Demeiry ${ }^{\mathrm{a}, \mathrm{b}}$, Ahmed Ali $^{\mathrm{b}, \mathrm{c}}$, Yasmine Abouleila ${ }^{\mathrm{b}, \mathrm{c}}$, Yoshihiro Shimizu ${ }^{\mathrm{d}}$, Tsutomu Masujima ${ }^{\mathrm{b} \dagger}$, \\ Randa Abdel Salam ${ }^{\mathrm{e}}$, Ghada Hadad ${ }^{\mathrm{e}}$, Samy Emara ${ }^{\mathrm{a}^{*}}$. \\ ${ }^{a}$ Faculty of Pharmacy, Misr International University (MIU), Km 28 Ismailia Road, Cairo, Egypt, ${ }^{b}$ Laboratory \\ for Single Cell Mass Spectrometry, Center for Biosystems Dynamics Research (BDR), RIKEN, 6-2-3 Furuedai, \\ Suita, Osaka 565-0874, Japan. ${ }^{c}$ Research Center, Misr International University, Km 28 Ismailia Road, Cairo, \\ Egypt. ${ }^{d}$ Laboratory for Cell-Free Protein Synthesis, Center for Biosystems Dynamics Research (BDR), RIKEN, \\ 6-2-3 Furuedai, Suita, Osaka 565-0874, Japan, ${ }^{e}$ Faculty of Pharmacy, Suez Canal University, Ismailia 41522, \\ Egypt.
}

Received on: 20. 07.2020

Revised on: 04. 09. 2020

Accepted on: 12. 09. 2020

Correspondence Author:

Tel:+201224537337

E-mail address:

mennademeiry@hotmail.com

\section{Abstract}

Ciprofloxacin belongs to the fluoroquinolone family, which exhibits biological activity against both gram-positive and gram-negative bacteria. The previously mentioned drug is of utmost importance due to the high incidence of resistance caused by other antibiotics. Thus, numerous research studies has been conducted to determine Ciprofloxacin as well as its metabolites owing to their contribution in the drug's biological activity. Liquid chromatography, gas chromatography, capillary electrophoresis and other separation techniques were used to develop, adapt and implement analytical techniques for the drug analysis. Other studies has made use of the hyphenation between liquid chromatography and mass spectrometry for Ciprofloxacin assay for quality control, biological fluids, animal tissues and numerous sample types. The recent advances in the ionization methods has led to the use of direct mass spectrometry techniques to analyze various sample types. This mini review aims to focus on the various analytical techniques and recent technologies implemented for Ciprofloxacin analysis.

Keywords: Ciprofloxacin; nanospray capillary; ion source; direct nanoelectrospray ionization-; targeted detection.

\section{Introduction}

Ciprofloxacin is a broad-spectrum antimicrobial agent, which falls under the fluoroquinolones class. The drug is beneficial as it acts against several infectious diseases where urinary tract infections are the most common ones (Vance-Bryan et al.,1990). Ciprofloxacin is effective against both gram negative and gram-positive bacteria (gram negative in particular), the mechanism of action targets topoisomerase IV inhibition. Unlike most antibiotics (penicillins, cephalosporins and aminoglycosides), ciprofloxacin is the least resistant antibiotic compared to the ones mentioned above (Navarro et al.,2002).

The drug is partially metabolized in the liver, giving four active metabolites; des-ethylene ciprofloxacin, oxo-ciprofloxacin, sulfo- 
ciprofloxacin and $\mathrm{N}$-acetyl ciprofloxacin, where oxo-Ciprofloxacin is the primary urinary metabolite and sulfo-Ciprofloxacin is the fecal metabolite (Vance-Bryanet al.,1990). A large percentage of the drug is excreted unchanged in the urine as well as all four metabolites (Eshar et al.,2018).

Accounting to the importance and the utmost need for such drug, several studies have employed various analytical techniques in owe of Ciprofloxacin analysis along with its metabolites; due to their major biological activity (Vance-Bryanet al.,1990).

Numerous techniques have been carried to analyse ciprofloxacin in various pharmaceutical dosage forms, for the purposes of quality control assays and method optimization. Other techniques have been developed for the drug analysis as well as its metabolites in biological fluids; urine, plasma, serum and saliva. However, when it comes to biological fluids, sample pre-treatment and method optimization are mandatory due to the complexity and endogenous components of such matrices (Esharet al.,2018, Vance-Bryanet al.,1990).

\section{Method of analysis}

\subsection{Liquid chromatography}

Numerous Liquid chromatographic (LC-MS) techniques have been designed to determine and quantitate Ciprofloxacin in various pharmaceutical dosage forms using ultraviolet (UV) and fluorescence detection systems. The chromatographic system has been the go to tool of analysis when it comes to mixtures, however such golden tool comes with a great deal of parameters to adjust and optimize in order to make use of its sensitivity; for instance the method flow rate, the mobile phase composition and $\mathrm{PH}$, the column, and the detector wavelength.

Several methods were developed to analyze and quantitate Ciprofloxacin in pharmaceutical dosage forms; Kassab et al, developed a technique to determine the drug in question in two pharmaceutical dosage forms using a mobile phase of water: acetonitrile: trimethylamine (80:20:0.3v/v/v) with $\mathrm{pH} 3.3$ at $279 \mathrm{~nm}$, UV detection (Kassab et al.,2005). Predrag et al, validated an analytical method for quality control assay of Ciprofloxacin with mobile phase of acetonitrile: water $(14: 86 \mathrm{v} / \mathrm{v}), \mathrm{pH} 3$ at $278 \mathrm{~nm}$ (Sibinović et al.,2005).
Others were concerned with the analysis of the targeted drug in biological fluids; analysis of human plasma to determine ciprofloxacin with an HPLCUV technique, prior to protein precipitation using acetonitrile at $277 \mathrm{~nm}$ (Vella et al.,2015). Plasma and urine samples assay using an isocratic RPHPLC technique with UV detection at $280 \mathrm{~nm}$; where the plasma samples were pretreated using acetonitrile and centrifuged to get rid of the proteins, while the urine samples were diluted with distilled water (Kamberi et al.,1998). Another HPLC-UV assay for Ciprofloxacin determination in human plasma following protein precipitation at 280nm was carried (Maya et al.,2001).

While several articles focused on Ciprofloxacin analysis using UV detection, others have utilized the fluorescent properties of the drug in question for analysis; an HPLC technique has been developed and validated for Ciprofloxacin assay in human plasma post excitation at $278 \mathrm{~nm}$, where protein precipitation was carried prior to analysis (Imre et al.,2003). Another study was carried by employing an isocratic HPLC assay of ciprofloxacin inaddition to three of the drug's metabolites in serum and diluted urine samples (Krol et al.,1995). Furthermore, an assay was done to analyze rabbit and human serum to quantitate Ciprofloxacin after excitation at $\lambda_{\text {exc }} 274 \mathrm{~nm}$ and emission at $\lambda_{\text {emi }} 418$ nm (Pei et al.,1994) .

\subsection{Capillary electrophoresis (CE)}

While most of the separation techniques used to analyze Ciprofloxacin are dependent on Liquid chromatography, some studies implemented Capillary electrophoresis (CE) as an alternative; due to the lesser amounts of organic solvents needed, the feasibility, safety and shorter run times. Michalska et al. developed and validated a $\mathrm{CE}$ technique to determine Ciprofloxacin and its impurities simultaneously (Michalska et al.,2004). Other studies applied CE to analyze drugs in biological matrices; Hernandez et al. carried an isotachophoresis-capillary zone electrophoretic technique to determine three quinolones in pig plasma samples, where a solid phase extraction step prior to analysis, was done as sample pretreatment (Hernández et al.,2002). Another biological study to determine two fluoroquinolones was proposed; Barron et al. implemented a solid-phase extraction procedure for chicken muscles, followed by a capillary electrophoretic method to determine Enrofloxacin and Ciprofloxacin (Barrón et al.,2001). 


\subsection{Spectrophotometry detection}

Numerous studies have utilized the spectrophotometry technique to analyze ciprofloxacin in mixtures along with other drugs; determination of Ciprofloxacin using a solid phase extraction (SPE) procedure followed by spectrophotometric analysis using UV detection (Pascual-Reguera et al.,2004). A quality control assay using a zero order spectrophotometry to analyze Ciprofloxacin in a mixture with Metronidazole was carried (Attia et al.,2016). Another quality control application was done to determine Ciprofloxacin in a mixture with Tinidazole using UV spectrophotometry (Bhalerao and Rote, 2012).

\subsection{Spectrofluorometric detection}

Ciprofloxacin is characterized by its high native fluorescence, which led to the development of several studies in this area. Navalon et al. developed a solid-phase spectrofluorometric technique to analyze residual amounts of Ciprofloxacin in human urine specimen (Navalón et al.,2000). Tatar proposed a fast, specific and sensitive spectrofluorometric method to analyze Ciprofloxacin along with three other fluoroquinolones in pharmaceutical dosage forms (Ulu, 2009).

\subsection{Mass spectrometry detection}

Mass spectrometry (MS) is one of the most recent powerful tools in the analytical field; such tool has given many privileges in terms of sensitivity and selectivity. Where the mass spectrometer has allowed determining the exact mass of an atom or a molecule, ionization of almost any compound being analyzed and consequently, ion fragmentation that allowed the interpretation of the resulting fragments. Thus, structural information regarding the targeted compound could be obtained. The many ionization techniques discovered have widened the scope of efficiency of the MS, which allowed the ionization of non-volatile complex compounds (e.g. proteins and polysaccharides) (De Hoffmann, 2000) .

\subsubsection{Liquid chromatography mass spectrometry (LC-MS)}

The hyphenation between the resolving power of liquid chromatography (LC) and the sensitivity of MS has led to the LC-MS technique, which evidently became the tool of choice when it comes to chromatography. Where the liquid chromatography separates the liquid phase and coverts it to gas and the MS ionizes the gas phase resulting into ions, which are fed into the MS detector for subsequent separation and analysis. LC-MS has proven superior to numerous techniques such as Gas chromatography mass spectrometry (GC-MS) because it is suitable for the analysis of non-volatile, thermally unstable and large ionic compounds (Packard and Primer,2017). Several studies adapted the LC-MS technique to determine Ciprofloxacin in various matrices and dosage forms; Wastewater samples were analyzed using LC-MS procedure with fluorescence detection to determine several fluoroquinolones including Ciprofloxacin at $\lambda_{\text {exc }} 278 \mathrm{~nm}$ and $\lambda_{\text {emi }}$ 445nm (Nakata et al.,2005). Another LC-MS technique was developed to determine the drug in question, where a double step SPE procedure was done prior to the assay (Caro et al.,2006). The use of such combined technique has given the analytical process an edge; where tandem mass spectrometry (LC-MS/MS) was used to elucidate the structure of the targeted compound in a mixture with Enorfloxacin in chicken tissues following a SPE step (Ferrari et al.,2015). Other numerous LCMS/MS techniques have been developed and adapted to analyze Ciprofloxacin in mixtures with other quinolones in food samples and meat products (Hatano,2004, Lee et al.,2013).

\subsubsection{Direct Electrospray ionization mass spectrometry (ESI-MS)}

The typical mass spectrometer consists of an ion source, a mass analyzer, a detector and a computer device for data display, and while all components are of great importance, the ionization technique has a huge impact on the process in terms of sensitivity and ionization efficiency. The recent advances in ionization technologies has allowed direct analysis of intact samples and subsequently shortcutting the separation step (chromatography), which minimized sample pretreatment, carryover effect accompanied by the chromatography system and most importantly less run times (Klampfl and Himmelsbach,2015). Ester caro etal, adapted the direct ESI-MS technique to determine Ciprofloxacin after a two-step SPE pretreatment. Which greatly reduced the matrix effect threatened by the numerous endogenous compounds present in the urine samples analyzed, and subsequently allowed direct analysis and evidently less run time (Caroet al.,2006)

\subsubsection{Direct Nano-electrospray ionization Mass spectrometry (nano-ESI-MS)}

Further advances in the mass spectrometry ionization techniques has led to the evolution of the 
standard ESI source into nano-ESI technology. While the typical ESI source has proven efficient and sensitive, Nano-ESI technology has a few extra remarkable features. Minute sample volumes are needed for analysis which makes it very suitable when it comes to limited volume samples, much lower flow rates and higher ionization efficiency, more stable spraying, smaller sizes of the initial droplets and subsequently less analyte amount lost (Karas et al.,2000).

Demeiry etal, adapted the direct nano-esi tandem mass spectrometry technique to identify and quantitate Ciprofloxacin in pharmaceutical dosage form as well as human urine samples (El Demeiry et al.,2020).

\section{Conclusion}

Fluoroquinolones are an important class of antibiotics that is active against numerous bacterial infections. Ciprofloxacin belongs to previously mentioned class; it is a broad-spectrum quinolonecarboxylic acid derivative, which is effective against gram-negative and positive bacteria (especially those resistant to other antibiotic classes like penicillins and cephalosporins). The drug is used to treat various respiratory tract infections as well as gastrointestinal disorders. The primary metabolism of the drug in question takes place in the liver resulting in four metabolites that contribute to the drug's biological activity. Thus, numerous studies and articles have developed and adapted several analytical techniques to determine and analyze Ciprofloxacin as well as its metabolites. A lot of research was carried to analyze dosage forms and pharmaceutical preparations. Other research was for the purpose of biological fluids and matrices assay of the drug in question, while some work was done as an adaptation and optimization of complex analytical techniques using Ciprofloxacin as the target analyte.

\section{References}

Attia KA, Nassar MW, El-Zeiny MB, Serag A. Zero order and signal processing spectrophotometric techniques applied for resolving interference of metronidazole with ciprofloxacin in their pharmaceutical dosage form. Spectrochimica Acta Part A: Molecular and Biomolecular Spectroscopy. 2016;154:232-6.

Barrón D, Jiménez-Lozano E, Cano J, Barbosa J. Determination of residues of enrofloxacin and its metabolite ciprofloxacin in biological materials by capillary electrophoresis. Journal of
Chromatography B: Biomedical Sciences and Applications. 2001;759(1):73-9.

Bhalerao SR, Rote AR. Application of UV spectrophotometric methods for estimation of ciprofloxacin and tinidazole in combined tablet dosage form. Int J Pharm Pharm Sci. 2012;4:464-7.

Caro E, Marcé RM, Cormack PA, Sherrington DC, Borrull F. Direct determination of ciprofloxacin by mass spectrometry after a two-step solid-phase extraction using a molecularly imprinted polymer. Journal of separation science. 2006;29(9):1230-6.

De Hoffmann E. Mass spectrometry. Kirk-Othmer Encyclopedia of Chemical Technology. 2000.

El Demeiry M, Ali A, Abouleila Y, Shimizu Y, Masujima T, Salam RA, et al. Quantification and targeted detection of ciprofloxacin in dosage form and human urine by direct injection nanoelectrospray ionization multi-stage mass spectrometry. Microchemical Journal. 2020;153:104534.

Eshar D, Wright LT, McCullough CE, Kukanich B. Pharmacokinetics of enrofloxacin and its metabolite ciprofloxacin following single-dose subcutaneous injection in black-tailed prairie dogs (Cynomys ludovicianus). American journal of veterinary research. 2018;79(6):658-63.

Ferrari SPG, Bonassa KP, Coelho MB, Ferreira CR, da Costa HF, Jara JLP, et al. High precision and selectivity for quantitation of enrofloxacin and ciprofloxacin in five chicken tissues using solid phase extraction and ESI LC-MS/MS for application in monitoring residues. Analytical Methods. 2015;7(7):3291-7.

Hatano K. Simultaneous determination of quinolones in foods by LC/MS/MS. Shokuhin eiseigaku zasshi Journal of the Food Hygienic Society of Japan. 2004;45(5):239-44.

Hernández M, Aguilar C, Borrull F, Calull M. Determination of ciprofloxacin, enrofloxacin and flumequine in pig plasma samples by capillary isotachophoresis-capillary zone electrophoresis. Journal of Chromatography B. 2002;772(1):163-72.

Imre S, Dogaru MT, Vari C, Muntean T, Kelemen L. Validation of an HPLC method for the determination of ciprofloxacin in human plasma. 
Journal of pharmaceutical and biomedical analysis. 2003;33(1):125-30.

Kamberi M, Tsutsumi K, Kotegawa T, Nakamura $\mathrm{K}$, Nakano S. Determination of ciprofloxacin in plasma and urine by HPLC with ultraviolet detection. Clinical chemistry. 1998;44(6):1251-5.

Karas M, Bahr U, Dülcks T. Nano-electrospray ionization mass spectrometry: addressing analytical problems beyond routine. Fresenius' Journal of Analytical Chemistry. 2000;366(6):669-76.

Kassab NM, Singh AK, Kedor-Hackmam ERM, Santoro MIRM. Quantitative determination of ciprofloxacin and norfloxacin in pharmaceutical preparations by high performance liquid chromatography. Revista Brasileira de Ciências Farmacêuticas. 2005;41(4):507-13.

Klampfl CW, Himmelsbach M. Direct ionization methods in mass spectrometry: An overview. Analytica chimica acta. 2015;890:44-59.

Krol G, Beck G, Benham T. HPLC analysis of ciprofloxacin and ciprofloxacin metabolites in body fluids. Journal of pharmaceutical and biomedical analysis. 1995;14(1-2):181-90.

Lee S, Kim B, Kim J. Development of isotope dilution-liquid chromatography tandem mass spectrometry for the accurate determination of fluoroquinolones in animal meat products: Optimization of chromatographic separation for eliminating matrix effects on isotope ratio measurements. Journal of Chromatography A. 2013;1277:35-41.

Maya MT, Gonçalves NJ, Silva NB, Morais JA. Simple high-performance liquid chromatographic assay for the determination of ciprofloxacin in human plasma with ultraviolet detection. Journal of Chromatography B: Biomedical Sciences and Applications. 2001;755(1-2):305-9.

Michalska K, Pajchel G, Tyski S. Determination of ciprofloxacin and its impurities by capillary zone electrophoresis. Journal of Chromatography A. 2004;1051(1):267-72.

Nakata H, Kannan K, Jones PD, Giesy JP. Determination of fluoroquinolone antibiotics in wastewater effluents by liquid chromatographymass spectrometry and fluorescence detection. Chemosphere. 2005;58(6):759-66.
Navalón A, Ballesteros O, Blanc R, Vílchez JL. Determination of ciprofloxacin in human urine and serum samples by solid-phase spectrofluorimetry. Talanta. 2000;52(5):845-52.

Navarro MDS, Milano CC, Castañeda AZ, Marinero MLS, Sánchez-Navarro A. Pharmacokinetics of ciprofloxacin as a tool to optimise dosage schedules in community patients. Clinical pharmacokinetics. 2002;41(14):1213-20.

Packard H, Primer A. Basics of LC/MS. 2017.

Pascual-Reguera MI, Parras GP, Díaz AM. Solidphase UV spectrophotometric method for determination of ciprofloxacin. Microchemical journal. 2004;77(1):79-84.

Pei Y, Meng X, Nightingale $\mathrm{CH}$. An improved HPLC assay for ciprofloxacin in biological samples. Zhongguo yao li xue bao $=$ Acta pharmacologica Sinica. 1994;15(3):197-201.

Sibinović P, Šmelcerović A, Palić R, Đorđević S, Marinković V. Ruggedness testing of an HPLC method for the determination of ciprofloxacin. Journal of the Serbian Chemical Society. 2005;70(7):979-86.

Ulu ST. Spectrofluorimetric determination of fluoroquinolones in pharmaceutical preparations. Spectrochimica Acta Part A: Molecular and Biomolecular Spectroscopy. 2009;72(1):138-43.

Vance-Bryan K, Guay DRP, Rotschafer JC. Clinical Pharmacokinetics of Ciprofloxacin. Clinical Pharmacokinetics. 1990;19(6):434-61.

Vella J, Busuttil F, Bartolo NS, Sammut C, Ferrito $\mathrm{V}$, Serracino-Inglott A, et al. A simple HPLC-UV method for the determination of ciprofloxacin in human plasma. Journal of Chromatography B. 2015;989:80-5. 